\title{
Survival and time to initiation of adjuvant chemotherapy among breast cancer patients: a systematic review and meta-analysis
}

\author{
Qiao-Hui Zhan ${ }^{1, *}$, Jian-Qin Fu' ${ }^{1, *}$, Fang-Meng Fu ${ }^{1}$, Jie Zhang ${ }^{1}$ and Chuan Wang ${ }^{1}$ \\ ${ }^{1}$ Department of General Surgery, Fujian Medical University Union Hospital, Fujian Province, Fuzhou, China \\ *These authors contributed equally to this work and should be considered as co-first authors \\ Correspondence to: Chuan Wang, email: chuanwang1968@yahoo.com \\ Keywords: time; adjuvant chemotherapy; breast cancer; suvival; meta-analysis \\ Received: August 01,2017 Accepted: November 17, $2017 \quad$ Published: December 07, 2017 \\ Copyright: Zhan et al. This is an open-access article distributed under the terms of the Creative Commons Attribution License 3.0 \\ (CC BY 3.0), which permits unrestricted use, distribution, and reproduction in any medium, provided the original author and source \\ are credited.
}

\section{ABSTRACT}

The relationship between survival and time to the start of adjuvant chemotherapy (AC) among breast cancer patients is unclear. In order to illustrate the effect of delaying the initiation of AC on survival we have undertaken a systematic review and meta-analysis.

We identified 12 available studies in the meta-analysis including 15 independent analytical groups. This meta-analysis showed that a 4-week delay before AC was associated with a significantly worse overall survival (OS)(HR=1.13; 95\% confidence interval [CI], 1.08-1.19) and disease free survival (DFS)(HR=1.14; 95\%CI, 1.051.24). Two studies categorized patients into hormone receptor-positive, ERBB2positive, and triple-negative breast cancer (TNBC) patients according to the clinicopathological features of breast cancer. The HRs for OS between waiting time (WT) $\leq 30$ days and 31-60 days in the subgroups were extracted and analyzed. The analysis demonstrated that a WT of 31-60 days was related to worse OS among patients with TNBC (HR, 1.26; 95\% CI, 1.08-1.48), but had no significant effect on OS among those with hormone receptor-positive (HR, 1.02; 95\% CI, 0.89-1.15) or ERBB2-postive (HR, 0.95; 95\%CI, 0.79-1.14) tumors.

In this meta-analysis of the eligible literatures reviewing the time to AC, a longer waiting time to adjuvant chemotherapy may lead to worse survival in breast cancer patients, especially in TNBC patients.

\section{INTRODUCTION}

In recent decades, the incidence of breast cancer has gradually increased and breast cancer has become the top killer of women [1]. Many randomized trials have demonstrated that breast cancer patients obtaine survival benefits from adjuvant chemotherapy [2]. Compared to patients who do not receive chemotherapy, adjuvant chemotherapy decreases by $30 \%$ to $40 \%$ the risk of breast cancer mortality [3]. Adjuvant chemotherapy is commonly used to improve survival and to reduce the risk of recurrence in breast cancer patients, particularly in patients with primary tumors that are large, estrogen receptor (ER) negative, high grade, and with lymph nodes involvement [4]. Most breast cancer patients start adjuvant chemotherapy within a few weeks after surgery, but it is still unclear whether a delay in the initiation of chemotherapy will lead to adverse outcomes. There are reasons to believe that early initiation of chemotherapy after surgery might improve survival, it has been known since the late 1970s that surgical trauma and tumor removal might result in an increased number of circulating tumor cells and an accelerated growth of micrometastases [5]. Clinically, the majority of breast cancer patients will eventually receive $\mathrm{AC}$, however, the optimal time to initiate AC is undefined. Some clinicians have suggested that a 3-month delay in time to start AC seems to be associated with a substantial decrease in the efficacy of systemic therapy [6]. Some studies have researched the effect of delays in initiating AC after breast cancer surgery. Four of these studies reported no association between initiation of $\mathrm{AC}$ and survival [7-10]. However, nine other 
studies found worse survival (disease free and/or overall) in patients starting AC more than four weeks [11], 35 days $[12,13], 60$ days [14], 10 weeks [15], 12-24 weeks [16], three months [17-19], and five months [20] after surgery. In order to explore the impact of delaying the initiation of AC on the survival of breast cancer patients, we have performed a systematic review of all the relevant studies and undertaken a meta-analysis of the available literature.

\section{METHODS}

\section{Searches}

Figure 1 presents the detailed procedures of the literature search and screening. All of the potentially relevant literature in PubMed, Google Scholar, EMBASE, Cochrane Database, and Web-of-Science from January-11978 to July-31, 2016 was searched using the key words; timing or time and adjuvant and chemotherapy or chemotherapeutic and breast cancer and survival. To yield more relevant articles, abstracts available from the online proceeding were searched for newly completed articles, especially those of annual meetings of the American Society of Clinical Oncology (ASCO) from 2007 to 2016. Furthermore, we reviewed the reference lists from relevant studies to identify studies not identified in the original search. The basic procedure of the metaanalysis was performed as previously described, and the procedure adheres to the standards of quality for reporting meta-analysis [21-23]. In order to reduce the effect of any publication bias, full-text articles and meeting abstracts, were eligible for inclusion.

\section{Selection}

All eligible literatures were required to satisfy the following criteria:

1. All of the breast cancer patients were treated with $\mathrm{AC}$, and the time interval after surgery and before beginning AC was documented.

2. The relationship between the time between surgery and starting AC and the subsequent outcome of breast cancer patients was reported.

3. Disease-free survival (DFS), relapse-free survival (RFS), event-free survival (EFS), or overall survival (OS) was used as the outcome for breast cancer patients. The hazard ratio (HR) of DFS, EFS, RFS, or OS with $95 \%$ confidence intervals (CIs) when they were reported or when there was sufficient data to calculate these values.

4. To reduce the impact of confounding factors between comparison groups, articles included in the final study cohort were identified to satisfy the following criteria: (1) the relevant prognostic factors were sufficiently described between compared groups; (2) either the compared groups or the reported results were balanced for the relevant prognostic factors [21].

\section{Exclusion criteria}

1. Studies were excluded by using nonstandard forms of AC. For instance: neoadjuvant chemotherapy, dose-dense chemotherapy or perioperative chemotherapy.

2. Studies that examined the impact of additional adjuvant therapies (e.g, radiotherapy or endocrine therapy) were excluded.

\section{Data extraction and conversion}

According to the procedure described previously $[21,23,24]$, we performed this step with several modifications. An HR for two survival measures (OS and/or DFS) was used to measure the effect in all studies. Since the definition of EFS and RFS was similar to DFS, EFS and RFS were treated as DFS for most studies. The representations of Waiting Time (WT) were categorized disparately in the available studies. In order to provide a common representation for evaluation of the results of individual studies, the WT effect was converted to a regression coefficient $(\beta)$ and its standard error (SE) corresponding to a continuous representation per 4-weeks of WT. In our study, 4-weeks of WT might be the most suitable cut-off time. Since there is a lack of original data to calculate the sensitivity and specificity, we could not use a ROC curve to evaluate the best cut-off. Most eligible studies used 4-weeks as cut-off. Four of nine studies were based on a cut-off of 4-weeks for OS, as were $3 / 5$ studies for DFS in this meta-analysis. Moreover, a one week delay would lead to a very small impact on survival which could not be detected. And when the cutoff time was extended to 4-weeks, an obvious effect on survival of delayed AC could be found. Therefore, we chose 4-weeks as the cut-off for the meta-analysis. Each WT category assigned a central value in each study. For studies with 2 WT groups, the two groups were defined as "before n weeks" and "after n weeks", thus we treated the reference time level as $n / 2$ weeks and the exposure time level as $n / 2+n$ weeks. The weekly $\beta$ was calculated as $\ln (\mathrm{HR}) /(\mathrm{Xn}-\mathrm{X} 0)$, and the corresponding $\mathrm{SE}$ of $\beta$ was calculated as $(\ln [$ upper of $95 \% \mathrm{CI}]-\ln [$ lower of $95 \% \mathrm{CI}]) /$ $([\mathrm{Xn}-\mathrm{X} 0] \times 3.92)$; where CI is the confidence interval, Xn denotes the exposure at group $\mathrm{n}$ level, and $\mathrm{X} 0$ denotes the exposure of the reference group. All the time units (days, weeks, or months) were transformed to "weeks" and "n" in the $\mathrm{Xn}$ denotes the number of weeks. If only a $P$-value was provided, the SE was calculated with the "test-based" method; $\mathrm{SE}=(\ln [\mathrm{HR}]) / \mathrm{Zp}$, where $Z p$ is the value of a unit-normal test (e.g., $Z p=1.96$ when $P=0.05$, 2-tailed test). For the studies with more than two WT groups, weighted least-squares linear regression of the $\ln (\mathrm{HR})$ at every exposure level in a study was used to estimate the summary $\beta$ with weights equal to the inverse of the variance of the HR estimates [25, 26]. The dependent variable for the regression was $\log (\mathrm{HR})$ corresponding to 
each study. The summary measures of HR per 4-weeks of delay from each study can be interpreted as the incidence rate ratio for death or recurrence with each 4-weeks of additional waiting for $\mathrm{AC}$, thus the summary measures presented here could be equal to e $\beta$ x 4 . These estimates are based on the assumption of a log linear relationship across WTs, and are only related to the range of WTs covered in the eligible studies.

\section{Study quality assessment}

Since all the articles included in our meta-analysis were nonrandomized, we use the 9-star Newcastle-Ottawa
Scale to assess the quality of eligible studies: The 9-star Newcastle-Ottawa Scale is used for assessing the quality of nonrandomized studies in meta-analysis.

\section{Meta-analysis}

The adjusted regression coefficients from individual studies were combined using a random-effects or fixed-effects model according to whether inter-study heterogeneity exists. We used the $\mathrm{Q}$ statistic and $\mathrm{I}^{2}$ to assess the between-studies heterogeneity [27]. A $P$-value $<0.05$ or $\mathrm{I}^{2}>25 \%$ meant heterogeneity [28]. The inverse variance was used to weight individual studies. The sensitivity

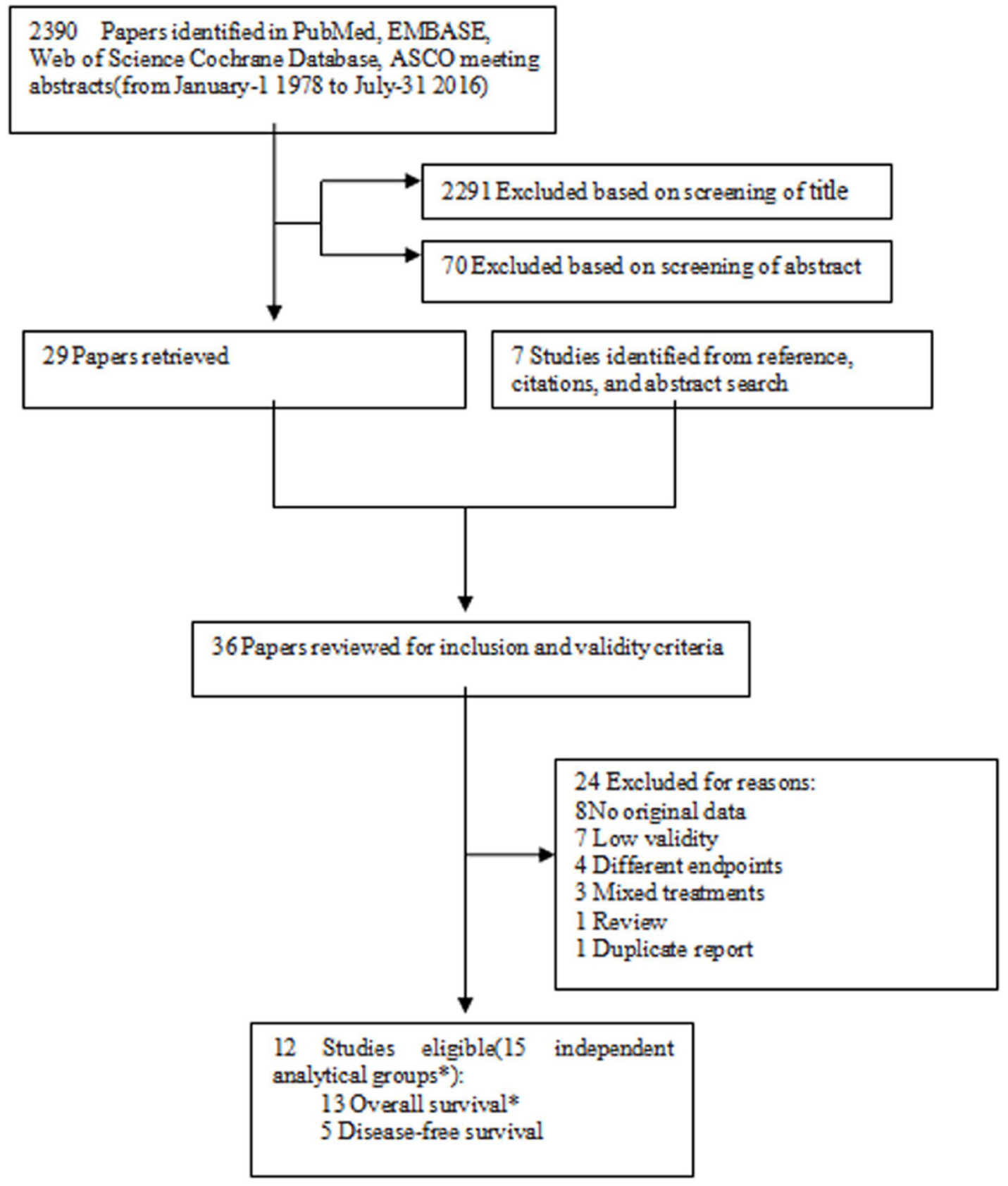

Figure 1: Flowchart of the study selection strategy. *Two studies include more than one analytical groups of overall survival. 
analysis was performed to find the potential outliers by sequentially omitting the largest studies and calculating a combined result from the remaining studies. The potential publication bias was detected in a funnel plot of $\log (\mathrm{HR})$ against its SE, and the degree of asymmetry was examined using Egger's test [29] $(P<0.05$ considered to be statistically significant). We performed all of the statistical analysis by using Stata 12.0 (Stata Corporation, College Station, TX) and SPSS20.0 (SPSS Inc, Chicago, IL). A two-tailed $P<0.05$ was considered statistically significant.

\section{RESULTS}

\section{Characteristics of selected studies}

The flow chart of the study selection strategy is shown in Figure 1. We selected 2,390 items published between 1989 and 2016 using the search strategy, and after reviewing their abstracts, 36 papers were potentially available. We further eliminated 24 reports which lacked data or did not meet the high validity criteria, and were left to consider 12 eligible papers including 78,462 breast cancer patients in our meta-analysis. One study was conducted prospectively [13], 3 studies [8, 12, 30] (with 5 analytical groups), were secondary analyses of randomized controlled trials, and the remaining 8 were retrospective investigations [14-19, 31] (9 analytical groups) using hospital-or population-based data (Table 1). Because two eligible publications included more than one analytical OS group, there were 13 independent analytical groups for OS and five for DFS in this meta-analysis (Table 2).

\section{Primary outcome: OS and DFS}

The study-specific waiting time categories and the HR results in the 13 analytical groups for OS are plotted in Figure 2A. The WTs were covered by the analytical groups, and arranged from 2 to 12 weeks. This figure indicates that the trend of the variation of HRs at different WTs in each study were similar, therefore, we can suppose the conversion of HRs from categories to an HR for a continuous representation by WT. Figure $2 \mathrm{~B}$ shows every single HR corresponding to the relative decrease in survival with each 4 -week increase in WT for each study. According to the different WT categories in every study, we used different methods to convert the HR estimates from the original studies to an HR per week of delay. For studies with two WT groups, the line was the same as that presented in Figure 2A. For studies using more than two categories, the HR was calculated by using meta-regression. The final HR used in our meta-analysis (HR per 4-weeks of delay) was represented by the 4-fold change of the slope of each line (by log converted HR) in Figure 2B. Figure $3 \mathrm{~A}$ is the forest plot of this meta-analysis for the OS and shows
HRs per 4-weeks of delay with $95 \%$ CIs for 13 analytical groups. The combined HR was 1.13 (95\%CI, 1.08-1.19) calculated by a random-effects model. There was a significant inter-study heterogeneity for OS $(P=0.00$; $I^{2}=78.9 \%$ ). In order to search the resource of heterogeneity, we undertook subgroup analysis according to the year that the studies were published (Figure 3B) and the between-study heterogeneity disappeared $\left(\mathrm{I}^{2}=0.0 \%\right)$. It demonstrated that the year was a major source of heterogeneity. Furthermore, we performed influence analysis (Figure 3C), which omits one study at a time and calculates the recombined HRs for the remainders, and the result showed no single study significantly influenced the pooled HR. However, we found that the HRs of two studies (the Cold-II study by Cold et al. [8] and the study by Ke-Da et al. [11]) obviously deviated from the combined HR. After excluding these two studies (Figure 3D) the between-study heterogeneity was significantly decreased $\left(P=0.06 ; \mathrm{I}^{2}=43.5 \%\right)$. It showed that the Cold-II and the study by Ke-Da were the other sources of heterogeneity. The funnel plot (Figure 4) and the Egger's test were also used to determine publication bias. There was no evidence of publication bias $(P>0.05)$ in this meta-analysis.

The same procedure of analysis was repeated for DFS, and the final forest plot for DFS is shown in Figure 5A. The combined HR was 1.09 (95\%CI, 1.031.14) calculated by a random-effects model. There was a significant heterogeneity between the included studies $\left(P=0.037, \mathrm{I}^{2}=60.9 \%\right)$. We also performed influence analysis for DFS. After omitting the Ke-Da et al. study [11] the inter-study heterogeneity disappeared (Figure $5 B)$. This indicated that the study by Ke-Da was the source of the heterogeneity

\section{Secondary outcome: the HRs for OS in different breast cancer subtypes}

Gagliato et al. [14] and Chavez-MacGregor et al. [17] categorized patients into hormone receptor-positive, ERBB2-positive and TNBC subgroups. The HRs for OS between WT $\leq 30$ days and 31-60 days in the three subgroups was extracted and analyzed. The combined $\mathrm{HR}$ in hormone receptor-positive and ERBB2-postive tumors were $1.02(95 \% \mathrm{CI}, 0.89-1.15)$ and $0.95(95 \% \mathrm{CI}$, 0.79-1.14), while the combined HR was 1.26 (95\%CI, 1.08-1.48) in TNBC patients (forest plot shown in Figure 6). All the above data were calculated by fixedeffects models. The funnel plot (Figure 7) showed no evidence of publication bias.

\section{DISCUSSION}

Adjuvant chemotherapy (AC) is one of the most important therapies for breast cancer patients. Nevertheless, the optimal time to initiate AC after surgery 
Table 1: Characteristics of eligible studies

\begin{tabular}{|c|c|c|c|c|c|c|c|c|c|c|}
\hline Source & $\begin{array}{l}\text { Place, Data type } \\
\text { and name }\end{array}$ & $\begin{array}{c}\text { Median age, } \\
\text { year }\end{array}$ & $\begin{array}{c}\text { Meno- pausal } \\
\text { status }\end{array}$ & Stage & $\begin{array}{c}\text { Hormone } \\
\text { receptor- } \\
\text { positive (\%) }\end{array}$ & Chemotherapy & $\begin{array}{c}\text { Median } \\
\text { FU }\end{array}$ & $\begin{array}{l}\text { Sample } \\
\text { size }\end{array}$ & $\begin{array}{l}\text { Study } \\
\text { qualiy" }^{*}\end{array}$ & $\begin{array}{l}\text { Adjustment } \\
\text { for covariates }\end{array}$ \\
\hline $\begin{array}{l}\text { Pronzato et al. } \\
\text { [13] } 1989\end{array}$ & Italy (Pros.) & $\begin{array}{c}51 \mathrm{yr} \\
\text { (range, 27-70) }\end{array}$ & Mixed & $\begin{array}{c}\text { Operable } \\
(\mathrm{LN}+)\end{array}$ & NR & CMF & $\begin{array}{c}37 \\
\text { months }\end{array}$ & 229 & 7 & $\begin{array}{c}\text { Age, nodes status, } \\
\text { menopausal status, } \\
\text { cycle number, } \\
\text { individual dose, intensity }\end{array}$ \\
\hline $\begin{array}{l}\text { Colleoni et al. } \\
\text { [30] } 2000\end{array}$ & $\begin{array}{l}\text { Multicenter } \\
\text { (CT, IBCSG) }\end{array}$ & $78 \%$ pts $\geq 40 \mathrm{yr}$ & Pre. & $\begin{array}{c}\text { Operable } \\
(\mathrm{LN}+)\end{array}$ & 87.4 & $\mathrm{CMF}$ & 7.7 years & 1,788 & 8 & $\begin{array}{l}\text { Age, size, nodal } \\
\text { status, vessel invasion, } \\
\text { and institution }\end{array}$ \\
\hline $\begin{array}{l}\text { Kerbrat et al. } \\
\text { [31] } 2005^{* *}\end{array}$ & $\begin{array}{c}\text { France } \\
\text { (Retros., FASG) }\end{array}$ & NR & NR & Operable & NR & Anthr--based & 9 years & 2,602 & 7 & $\begin{array}{l}\text { Multivariate, adjustmen; } \\
\text { adjusted factors not } \\
\text { reported }\end{array}$ \\
\hline $\begin{array}{l}\text { Cold et al. [8] } \\
\quad 2005 \text { (I) }\end{array}$ & $\begin{array}{c}\text { Denmark } \\
\text { (CT, DBCG) }\end{array}$ & $\begin{array}{c}53 \% \mathrm{pts}<46 \mathrm{yr} \\
43 \% \mathrm{pts} \\
46-55 \mathrm{yr} \\
3 \% \mathrm{pts}>55 \mathrm{yr}\end{array}$ & Mixed & Operable & 77 & Classical CMF & NR & 352 & 6 & $\begin{array}{l}\text { Age, tumour size, } \\
\text { nodes status, histological } \\
\text { type, grade, hormone } \\
\text { receptor status, and } \\
\text { adjuvant irradiation }\end{array}$ \\
\hline $\begin{array}{l}\text { Cold et al. [8] } \\
2005 \text { (II) }\end{array}$ & $\begin{array}{c}\text { Denmark } \\
\text { (CT, DBCG) }\end{array}$ & $\begin{array}{c}40 \% \text { pts }<46 \mathrm{yr} \\
40 \% \text { pts } 46- \\
55 \mathrm{yr} \\
20 \% \text { pts }>55 \mathrm{yr}\end{array}$ & Mixed & Operable & 58.3 & CMF i.v. & NR & 6,065 & 8 & $\begin{array}{l}\text { Age, tumour size, } \\
\text { nodes status, histological } \\
\text { type, grade, hormone } \\
\text { receptor status, and } \\
\text { adjuvant irradiation }\end{array}$ \\
\hline $\begin{array}{l}\text { Cold et al. [8] } \\
2005 \text { (III) }\end{array}$ & $\begin{array}{c}\text { Denmark } \\
\text { (CT, DBCG) }\end{array}$ & $\begin{array}{c}40 \% \text { pts }<46 \mathrm{yr} \\
40 \% \text { pts } 46- \\
55 \mathrm{yr} \\
20 \% \text { pts }>55 \mathrm{yr}\end{array}$ & Mixed & Operable & 61.8 & $\mathrm{CEF}$ & NR & 1,084 & 7 & $\begin{array}{l}\text { Age, tumour size, } \\
\text { nodes status, histological } \\
\text { type, grade, hormone } \\
\text { receptor status, and } \\
\text { adjuvant irradiation }\end{array}$ \\
\hline $\begin{array}{l}\text { Hershman et al. } \\
\quad \text { [18] } 2006\end{array}$ & $\begin{array}{c}\text { USA } \\
\text { (Retros., SEER) }\end{array}$ & $100 \% \mathrm{pts} \geq 65 \mathrm{yr}$ & Post. & I-II & 58 & Polychemotherapy & NR & 5,003 & 8 & $\begin{array}{l}\text { Age, race, live location, } \\
\text { stage, hormonereceptor, } \\
\text { grade, comorbid conditions, } \\
\text { SES score, marital status, } \\
\text { teaching hospital, surgery, } \\
\text { and radiation }\end{array}$ \\
\hline $\begin{array}{l}\text { Lohrisch et al. } \\
\text { [16] } 2006\end{array}$ & USA (Retros.,) & $47 \mathrm{yr}$ & Mixed & I-II & 55.9 & $\begin{array}{l}\text { CMF and Anthr.- } \\
\text { based }\end{array}$ & 6.2 years & 2,594 & 8 & $\begin{array}{l}\text { Age, size, nodal } \\
\text { status, lymphatic or } \\
\text { vascular invasion, } \\
\text { and anthracycline }\end{array}$ \\
\hline $\begin{array}{l}\text { Nurgalieva } \\
\quad \text { et al. } \\
\text { [19] } 2013\end{array}$ & $\begin{array}{c}\text { USA } \\
\text { (Retros., BCCA) }\end{array}$ & $100 \% \mathrm{Pts} \geq 65 \mathrm{yr}$ & Post. & I-III & NR & Polychemotherapy & NR & 14,380 & 8 & $\begin{array}{l}\text { Age, marriage status, } \\
\text { tumor stage, size, } \\
\text { grade, hormonereceptor } \\
\text { status, comorbidity, year of } \\
\text { diagnosis, SEER } \\
\text { region, primary surgery } \\
\text { radiotherapy, chemotherapy, } \\
\text { arace/ethnicity }\end{array}$ \\
\hline $\begin{array}{l}\text { Downing et al. } \\
\text { [15] 2014(I) }\end{array}$ & UK (Retros.) & $\begin{array}{l}27 \% \text { pts }<45 \mathrm{yr} \\
73 \% \mathrm{pts} \geq 45 \mathrm{yr}\end{array}$ & Mixed & I-III & NR & Polychemotherapy & NR & 6,100 & 8 & $\begin{array}{l}\text { age, stage, } \mathrm{RT} \\
\text { receive after } \mathrm{CT}, \\
\text { and year of treatment } \\
\text { comorbidity, surgery, } \\
\text { reconstruction }\end{array}$ \\
\hline $\begin{array}{l}\text { Downing et al. } \\
\text { [15] 2014(II) }\end{array}$ & UK (Retros.) & $\begin{array}{c}25.8 \% \mathrm{pts} \\
<45 \mathrm{yr} \\
74.2 \% \mathrm{pts} \\
\geq 45 \mathrm{yr}\end{array}$ & Mixed & I-III & NR & Polychemotherapy & NR & 4,266 & 8 & $\begin{array}{l}\text { age, stage, RT } \\
\text { receive after CT, and year } \\
\text { of treatment comorbidity, } \\
\text { surgery, reconstruction }\end{array}$ \\
\hline $\begin{array}{l}\text { Gagliato et al. } \\
\text { [14] } 2014\end{array}$ & USA (Retros.,) & $\begin{array}{c}50 \mathrm{yr} \\
\text { (range, 19-85) }\end{array}$ & Mixed & I-III & 65.4 & Polychemotherapy & $\begin{array}{c}59.3 \\
\text { months }\end{array}$ & 6,827 & 8 & $\begin{array}{l}\text { age, race/ethnicity, tumor } \\
\text { size nodal status grade, } \\
\text { LVI, type of surgery } \\
\text { comorbidity }\end{array}$ \\
\hline $\begin{array}{l}\text { Farolfi et al. } \\
\text { [12] } 2015\end{array}$ & $\begin{array}{c}\text { Italy (CT, } \\
\text { NCT01031030) }\end{array}$ & $\begin{array}{c}52 \mathrm{yr} \\
\text { (range, 26-70) }\end{array}$ & Mixed & Operable & 73.9 & $\begin{array}{c}\text { CMF-E, E-CMF } \\
\text { and CMF }\end{array}$ & $\begin{array}{c}105 \\
\text { months }\end{array}$ & 921 & 8 & $\begin{array}{c}\text { Nodal involvemt, } \\
\text { oestrogen recept HER2 } \\
\text { status; Ki67 value Type } \\
\text { of adjuvant chemotherapy, } \\
\text { menopausal status and } \\
\text { tumour size }\end{array}$ \\
\hline
\end{tabular}




\begin{tabular}{|c|c|c|c|c|c|c|c|c|c|c|}
\hline $\begin{array}{l}\text { Chavez et al. } \\
\text { [17] } 2015\end{array}$ & USA (Retros.) & $53 \mathrm{yr}$ & Mixed & I-III & NR & Polychemotherapy & 62.7 months & 24,843 & 8 & $\begin{array}{l}\text { age, sex, race/ethnicity, SES, } \\
\text { year of diagnosis, stage, subtype, } \\
\text { marital status, type of surgery, } \\
\text { primary payer reconstructive } \\
\text { surgery, whether treated at a } \\
\text { NCI-designated cancer center }\end{array}$ \\
\hline $\begin{array}{l}\text { Ke-Da et al. } \\
\text { [11] } 2016\end{array}$ & $\begin{array}{l}\text { China } \\
\text { (Retros.) }\end{array}$ & $50 \mathrm{yr}$ & Mixed & I-IIIa & 71.0 & $\begin{array}{l}\text { Anthr.-based } \\
\text { or Anthr.-/taxane- } \\
\text { based. }\end{array}$ & 72 months & 1408 & 8 & $\begin{array}{l}\text { age, tumor size, nodal status, } \\
\text { surgical modality, and endocrine } \\
\text { therapy }\end{array}$ \\
\hline $\begin{array}{l}\text { Farolfi et al. } \\
\text { [12] } 2015\end{array}$ & $\begin{array}{c}\text { Italy } \\
\text { (CT,NCT01031030) }\end{array}$ & $\begin{array}{c}52 \mathrm{yr} \\
\text { (range, 26-70) }\end{array}$ & Mixed & Operable & 73.9 & $\begin{array}{l}\text { CMF-E, E-CMF } \\
\text { and CMF }\end{array}$ & 105 months & 921 & 8 & $\begin{array}{c}\text { Nodal involvemt, } \\
\text { oestrogen recept } \\
\text { HER2 status; } \\
\text { Ki67 value } \\
\text { Type of adjuvant chemotherapy, } \\
\text { menopausal status and tumour } \\
\text { size }\end{array}$ \\
\hline $\begin{array}{l}\text { Chavez et al. } \\
\text { [17] } 2015\end{array}$ & USA (Retros.) & $53 \mathrm{yr}$ & Mixed & I-III & NR & Polychemotherapy & 62.7 months & 24,843 & 8 & $\begin{array}{l}\text { age, sex, race/ethnicity, } \\
\text { SES, year of diagnosis, stage, } \\
\text { subtype, marital status, } \\
\text { type of surgery, primary payer } \\
\text { reconstructive surgery, } \\
\text { whether treated at a NCI-- } \\
\text { designated cancer center }\end{array}$ \\
\hline $\begin{array}{l}\text { Ke-Da et al. } \\
\text { [11] } 2016\end{array}$ & $\begin{array}{l}\text { China } \\
\text { (Retros.) }\end{array}$ & $50 \mathrm{yr}$ & Mixed & I-IIIa & 71.0 & $\begin{array}{c}\text { Anthr.-based } \\
\text { or Anthr.-/taxane- } \\
\text { based. }\end{array}$ & 72 months & 1408 & 8 & $\begin{array}{l}\text { age, tumor size, nodal status, } \\
\text { surgical modality, and endocrine } \\
\text { therapy }\end{array}$ \\
\hline
\end{tabular}

Abbreviations: Anthr, Anthracycline; BCCA, British Columbia Cancer Agency; CMF, Cyclophosphamide, methotrexate and fluorouracil; CT, Clinical trial; DBCG, Dansh Breast Cancer Cooperative Group; FASG, French Adjuvant Study Group; FU, Follow up; IBCSG, International Breast Cancer Study Group; LN+, Lymphnodes positive; NR, Not reported; Post, Postmenopausal; Pros,

Prospectivestudy; Retro, Retrospectivestudy; SEER, The Surveillance, Epidemiology, and End-Resultsdatabase."Evaluated by the9-star Newcastle-Ottawa Scale.

"The publish type of this study is a meeting abstract.

is still unclear. Due to the potential ethical problems it is unlikely that a prospective clinical trial can be undertaken to explore the association between time delay to initiate $\mathrm{AC}$ and survival in breast cancer patients. Moreover, the published randomized controlled clinical trials do not directly suggest the time frame of $\mathrm{AC}$, and the time to initiate $\mathrm{AC}$ after surgery ranges from two to 12 weeks [32-35] in different trials. Therefore, the only way to perform our study is to rely on retrospective data. In this meta-analysis the results reveal that OS decreases by $13 \%$ and DFS decreases by $14 \%$ for every four weeks that AC

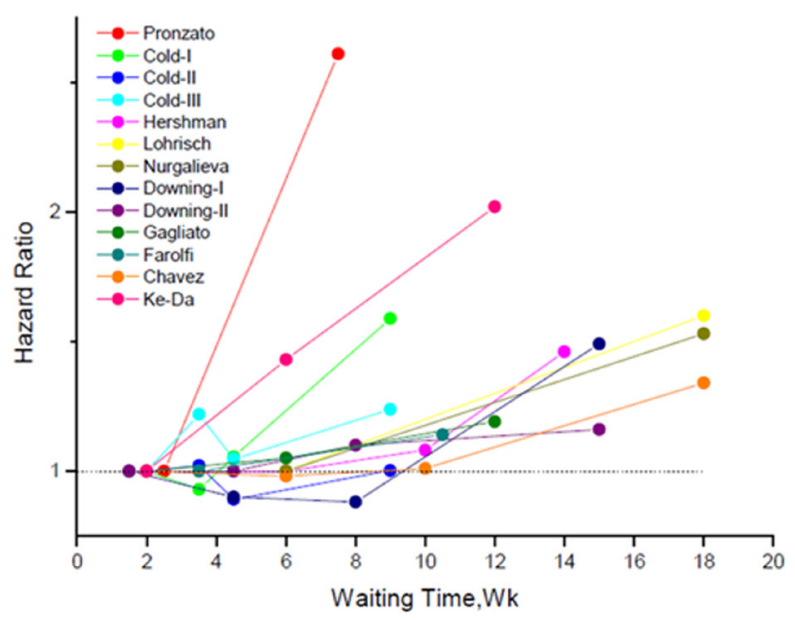

A

Figure 2: Individual hazard ratio for overall survival according to waiting time categories. (A) The relationship between waiting time categories and overall survival in the 12 independent analytical groups. The hazard ratio (HR) represents a comparison with the first waiting time category in each study (as reference). The first author of each study is shown. (B) Conversion of HR estimates from the original studies to an HR per week of delay. The slope of each line represents the change in the log HR per week of delay. The line for each individual study is located over the range of waiting times. The thick line indicates the weighted average of the HRs from the individual studies. The vertical axis is on a $\log$ scale. was delayed. Yu et al. [24] reported a similar result: OS decreases by $15 \%$ and DFS decreases by $16 \%$ for every four week delay in the initiation of AC.

Adjuvant chemotherapy decreases the risk of breast cancer mortality mainly through eradication of micrometastatic tumor deposits in breast cancer patients. Some clinical studies suggest that an AC delay to 12 weeks will significantly reduce the effectiveness of systemic therapy. The main theoretical controversies for adverse effects of treatment delay include mathematical models showings that drug resistance mutations can develop over time [36], and from mouse models showing

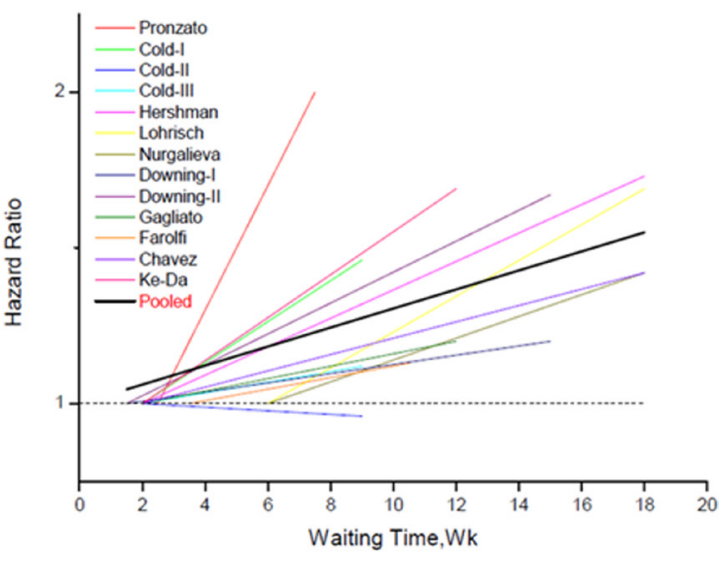

B 
Table 2: Study-specific waiting time categories and outcomes

\begin{tabular}{|c|c|c|c|c|}
\hline \multirow{2}{*}{ Source } & \multirow{2}{*}{$\begin{array}{c}\text { WT } \\
\text { categories }\end{array}$} & \multirow{2}{*}{$\begin{array}{c}\text { Sample } \\
\text { size }\end{array}$} & \multicolumn{2}{|c|}{ HR $(95 \% C I)$} \\
\hline & & & OS & DFS/RFS/EFS \\
\hline $\begin{array}{l}\text { Pronzato et al. } \\
\text { [13] } 1989\end{array}$ & $\begin{array}{l}\leq 35 \text { days } \\
>35 \text { days }\end{array}$ & $\begin{array}{l}116 \\
113\end{array}$ & $\begin{array}{c}\text { Reference } \\
2.61(1.26-5.39)\end{array}$ & - \\
\hline $\begin{array}{l}\text { Colleoni et al. } \\
\text { [30] } 2000\end{array}$ & $\begin{array}{l}<21 \text { days } \\
\geq 21 \text { days }\end{array}$ & $\begin{array}{c}599 \\
1,189\end{array}$ & - & $\begin{array}{c}0.88(0.76-1.03) \\
\text { Reference }\end{array}$ \\
\hline $\begin{array}{l}\text { Kerbrat, et al. } \\
\text { [31] } 2005\end{array}$ & $\begin{array}{l}<28 \text { days } \\
28-42 \text { days } \\
>42 \text { days }\end{array}$ & $\begin{array}{c}1,614 \\
883 \\
105\end{array}$ & - & $\begin{array}{c}0.85(0.65-1.05) \\
\text { Reference }\end{array}$ \\
\hline $\begin{array}{l}\text { Cold et al. } \\
\text { [8] } 2005 \text { (I) }\end{array}$ & $\begin{array}{l}1-3 \text { wks } \\
3-4 \text { wks } \\
4-5 \text { wks } \\
5-13 \text { wks }\end{array}$ & $\begin{array}{c}58 \\
92 \\
75 \\
127\end{array}$ & $\begin{array}{c}\text { Reference } \\
0.929(0.441-1.957) \\
1.549(0.761-3.149) \\
1.588(0.856-2.948)\end{array}$ & - \\
\hline $\begin{array}{l}\text { Cold et al. } \\
\text { [8] } 2005 \text { (II) }\end{array}$ & $\begin{array}{l}1-3 \text { wks } \\
3-4 \text { wks } \\
4-5 \text { wks } \\
5-13 \text { wks }\end{array}$ & $\begin{array}{l}1,509 \\
1,581 \\
1,423 \\
1,552\end{array}$ & $\begin{array}{c}\text { Reference } \\
1.021(0.903-1.155) \\
0.890(0.782-1.012) \\
1.002(0.884-1.136)\end{array}$ & - \\
\hline $\begin{array}{l}\text { Cold et al. } \\
\text { [8] } 2005 \text { (III) }\end{array}$ & $\begin{array}{l}1-3 \text { wks } \\
3-4 \text { wks } \\
4-5 \text { wks } \\
5-13 \text { wks }\end{array}$ & $\begin{array}{l}188 \\
305 \\
263 \\
328\end{array}$ & $\begin{array}{c}\text { Reference } \\
1.218(0.800-1.854) \\
1.045(0.716-1.525) \\
1.238(0.861-1.782)\end{array}$ & - \\
\hline $\begin{array}{l}\text { Hershman et al. } \\
\text { [18] } 2006\end{array}$ & $\begin{array}{l}<1 \text { month } \\
1-2 \text { months } \\
2-3 \text { months } \\
>3 \text { months }\end{array}$ & $\begin{array}{c}2,361 \\
1,846 \\
323 \\
477\end{array}$ & $\begin{array}{c}\text { Reference } \\
1.00(0.88-1.14) \\
1.08(0.85-1.36) \\
1.46(1.21-1.75)\end{array}$ & - \\
\hline $\begin{array}{l}\text { Lohrisch et al. } \\
\text { [16] } 2006\end{array}$ & $\begin{array}{c}\leq 4 \mathrm{wks} \\
4-8 \mathrm{wks} \\
8-12 \mathrm{wks} \\
12-24 \mathrm{wks}\end{array}$ & $\begin{array}{c}993 \\
1,272 \\
217 \\
112\end{array}$ & $\begin{array}{c}\text { Reference } \\
1.6(1.2-2.3)\end{array}$ & - \\
\hline $\begin{array}{l}\text { Nurgalieva et al. } \\
\text { [19] } 2013\end{array}$ & $\begin{array}{l}\leq 3 \text { months } \\
>3 \text { months }\end{array}$ & $\begin{array}{c}12,748 \\
1,632\end{array}$ & $\begin{array}{c}\text { Reference } \\
1.53(1.32-1.80)\end{array}$ & - \\
\hline $\begin{array}{l}\text { Downing et al. } \\
\text { [15] } 2014 \text { (I) }\end{array}$ & $\begin{array}{l}\leq 3 \mathrm{wks} \\
3-6 \mathrm{wks} \\
6-10 \mathrm{wks} \\
>10 \mathrm{wks}\end{array}$ & $\begin{array}{c}557 \\
3,253 \\
1,897 \\
393\end{array}$ & $\begin{array}{c}\text { Reference } \\
0.90(0.73-1.12) \\
0.88(0.70-1.10) \\
1.49(1.13-1.95)\end{array}$ & - \\
\hline $\begin{array}{l}\text { Downing et al. } \\
\text { [15] } 2014 \text { (II) }\end{array}$ & $\begin{array}{l}\leq 3 \mathrm{wks} \\
3-6 \mathrm{wks} \\
6-10 \mathrm{wks} \\
>10 \mathrm{wks}\end{array}$ & $\begin{array}{c}1,186 \\
2,279 \\
652 \\
149\end{array}$ & $\begin{array}{c}\text { Reference } \\
1.00(0.85-1.18) \\
1.10(0.88-1.37) \\
1.16(0.80-1.67)\end{array}$ & - \\
\hline $\begin{array}{l}\text { Gagliato et al. } \\
\text { [14] } 2014\end{array}$ & $\begin{array}{l}\leq 30 \text { days } \\
31-60 \text { days } \\
\geq 61 \text { days }\end{array}$ & $\begin{array}{l}2,716 \\
2,994 \\
1,117\end{array}$ & $\begin{array}{c}\text { Reference } \\
1.05(0.94-1.18) \\
1.19(1.02-1.38)\end{array}$ & $\begin{array}{c}\text { Reference } \\
1.04(0.94-1.14) \\
1.10(0.97-1.25)\end{array}$ \\
\hline $\begin{array}{l}\text { Farolfi et al. } \\
\text { [12] } 2015\end{array}$ & $\begin{array}{l}\leq 7 \mathrm{wks} \\
>7 \mathrm{wks}\end{array}$ & $\begin{array}{l}818 \\
103\end{array}$ & $\begin{array}{c}\text { Reference } \\
1.14(0.96-1.34)\end{array}$ & $\begin{array}{c}\text { Reference } \\
1.15(1.02-1.30)\end{array}$ \\
\hline $\begin{array}{l}\text { chavez et al. } \\
\text { [17] } 2015\end{array}$ & $\begin{array}{c}\leq 30 \text { days } \\
31-60 \text { days } \\
61-90 \text { days } \\
\geq 91 \text { days }\end{array}$ & $\begin{array}{c}5,224 \\
12,432 \\
4,765 \\
2,422\end{array}$ & $\begin{array}{c}\text { Reference } \\
0.98(0.87-1.09) \\
1.01(0.88-1.16) \\
1.34(1.15-1.57)\end{array}$ & - \\
\hline $\begin{array}{l}\text { Ke-Da et al. } \\
\text { [11] } 2016\end{array}$ & $\begin{array}{l}0-4 \mathrm{wks} \\
4-8 \mathrm{wks} \\
>8 \mathrm{wks}\end{array}$ & $\begin{array}{c}871 \\
446 \\
91\end{array}$ & $\begin{array}{c}\text { Reference } \\
1.43(0.94-2.19) \\
2.02(1.10-3.71)\end{array}$ & $\begin{array}{c}\text { Reference } \\
1.14(0.83-1.56) \\
1.86(1.19-2.90)\end{array}$ \\
\hline
\end{tabular}

Abbreviations: WT, Waiting time; HR, Hazard ratio; CI, Confidence interval; OS, Overall survival; DFS, Disease-free survival; RFS, Relapse-free survival. 

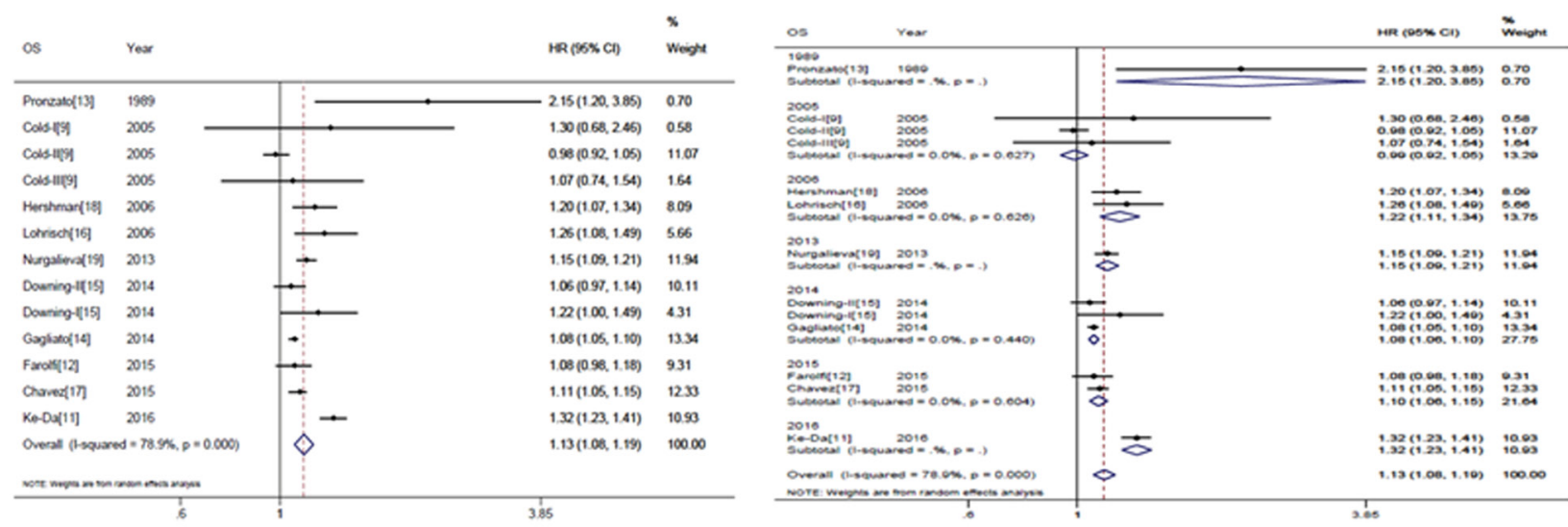

A

B
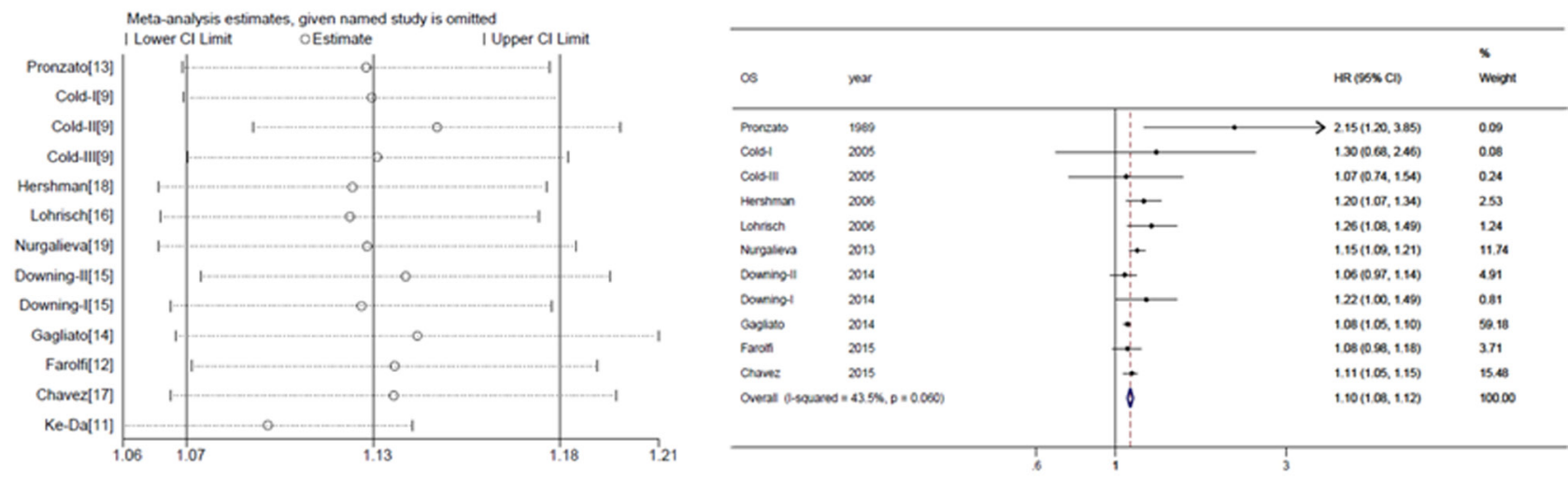

C

D

Figure 3: Individual study and overall hazard ratios of relationships between every 4-week delay in initiation of adjuvant chemotherapy and overall survival. (A) Shows individual study and overall hazard ratios (HR) per 4-weeks of delay with $95 \%$ confidence interval (CI) for OS. The size of each square is proportional to the weight of the study. For the combined result, the length of the diamond represents the $95 \% \mathrm{CI}$ of the summary. (B) Shows subgroup analysis for OS according to the year that the studies were published. (C) Shows the influence of individual studies on the pooled HR for OS. The vertical axis indicates the overall HR and the two vertical axes indicate its $95 \% \mathrm{CI}$. Every hollow round shape indicates the pooled OR when the left study is omitted in this meta-analysis. The two ends of every broken line represent the respective $95 \%$ CI. (D) Shows remaining studies after excluding Cold-II and Ke-Da studies; hazard ratios (HR) per 4-week of delay with 95\% confidence interval (CI) for OS.

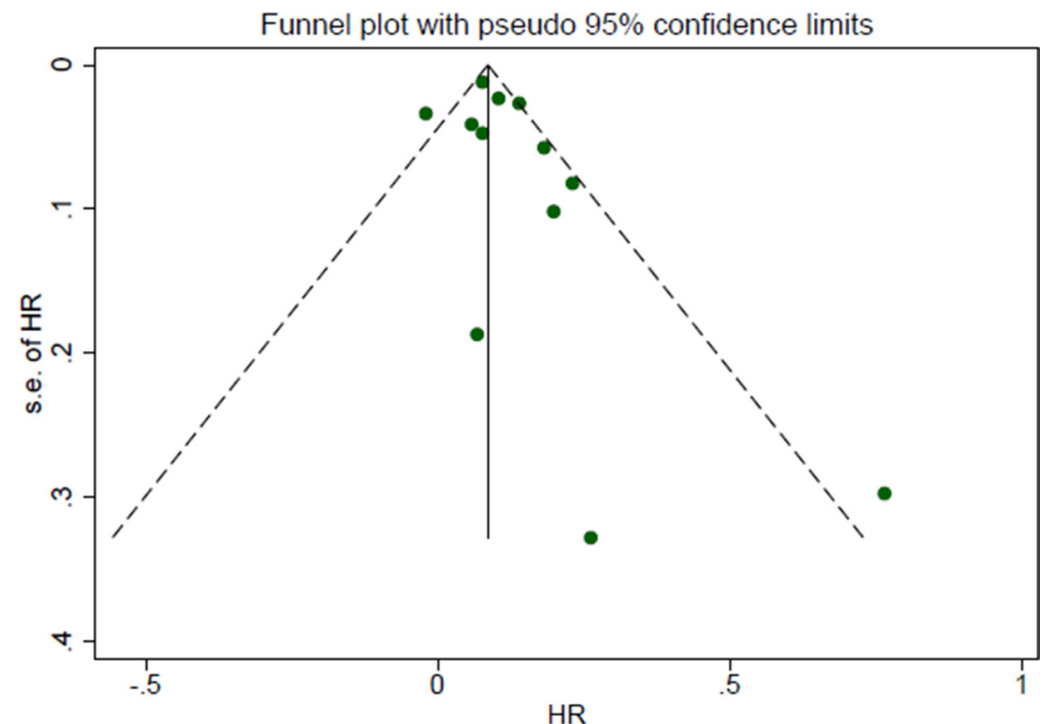

Figure 4: Funnel plot of the relationship between the hazard ratio and standard error of the log HR for overall survival. Hazard ratio (HR) estimates are the effect per 4 weeks of waiting time. The dotted line indicates the combined HR for all studies of overall survival. Filled circles represent the 12 studies to account for potential publication bias. 
accelerated micrometastatic tumor growth following primary tumor removal [37]. In addition, according to a recent understanding of the significant interplay between the immune system and tumor-produced factors, one could make the assumption that primary tumor removal may reinstate a patient's native antitumor immunity as a result of eliminating the primary source of tumor-mediated immune suppression.

The available studies of the association between survival and the time between surgery to and initiation of $\mathrm{AC}$ were included in our meta-analysis. OtheSomer

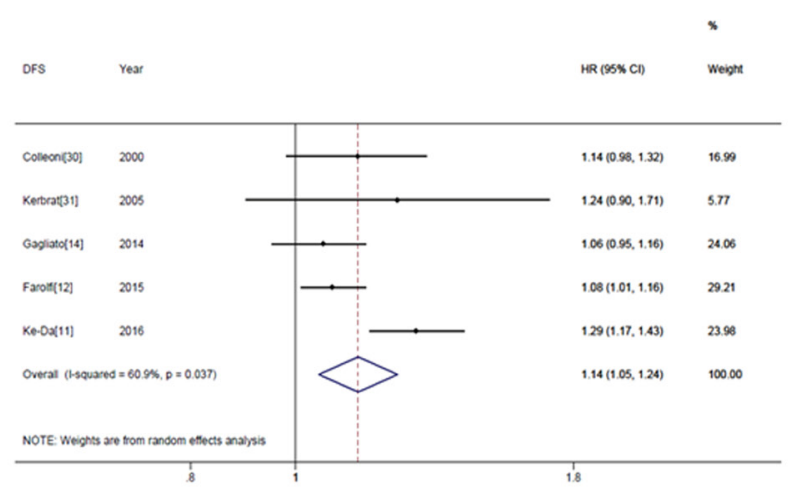

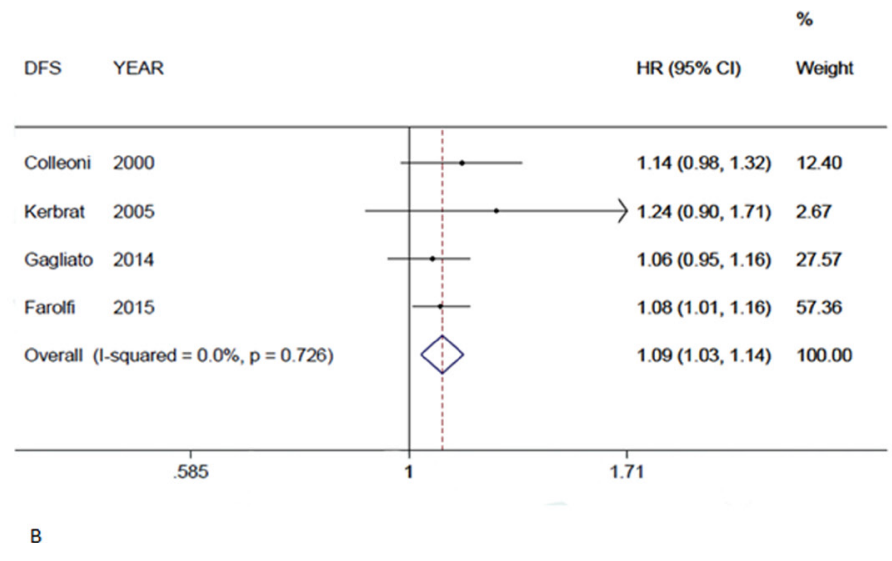

Figure 5: Individual study and overall hazard ratios of relationships between every 4-week delay in initiation of adjuvant chemotherapy and disease-free survival. (A) Individual and overall hazard ratios (HR) per 4-weeks of delay with 95\% confidence interval (CI) for DFS are shown. The size of each square is proportional to the weight of the study. For the combined result, the length of the diamond represents the $95 \%$ CI of the summary. (B) Shows remaining studies after excluding the Ke-Da study; hazard ratios (HR) per 4-week of delay with 95\% confidence interval (CI) for DFS.

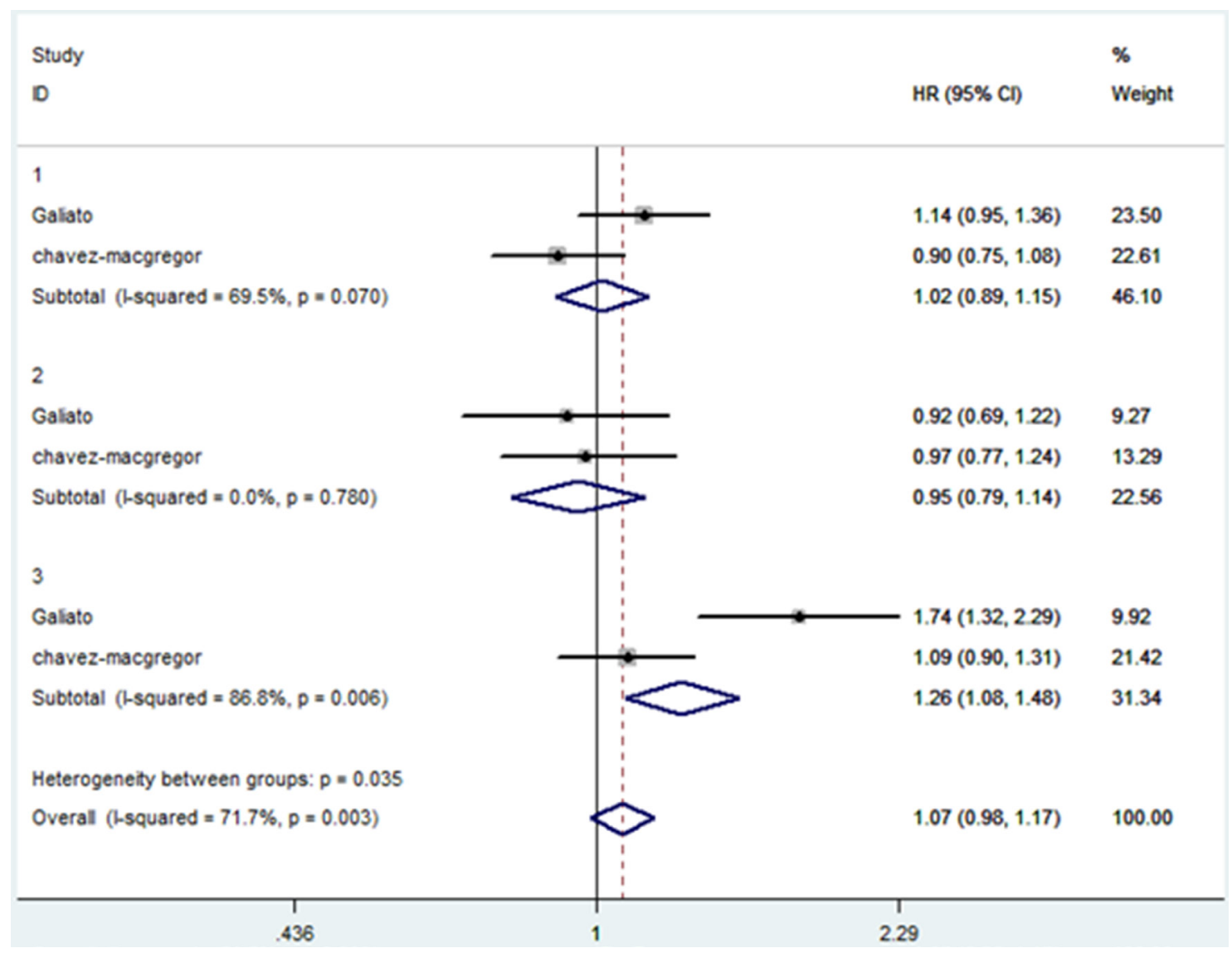

Figure 6: Comparison of overall survival between WT $\leq \mathbf{3 0}$ days and 31-60 days in the three subgroups. The size of each square is proportional to the weight of the study. For the combined result, the length of the diamond represents the $95 \% \mathrm{CI}$ of the summary. Numbers indicate different subgroups.1-hormone receptor-positive breast cancer, 2-ERBB2-positive breast cancer, 3-TNBC. 
relevant articles were excluded, because these studies were not up to the inclusive criteria. Alkis et al. [38], Brooks et al. [39] and another Turkish study [20] lacked sufficient data to calculate an adjusted and quantitative HR. Buzdar et al. [7], Sanchez et al. [9], Shannon et al. [10] and Samur et al. [40] did not show a worse outcome for patients with AC started later compared to those with AC stared early. A similar meta-analysis by Biagi et al. [41] indicated that a 4-week delay initiation of AC might lead to an obvious decrease in OS (HR $=1.06 ; 95 \%$ CI 1.02-1.10) and DFS $(\mathrm{HR}=1.08 ; 95 \%$ CI $1.03-1.14)$ in breast cancer. This study, however, was only an abstract, and it used a fixed-effect model to combine the individual researches studies although a significant heterogeneity between studies did exist.

This meta-analysis shows a detrimental effect on survival when delaying AC. Nevertheless, there was a significant inter-study heterogeneity for OS and DFS. To search for the source of heterogeneity, we performed a subgroup analysis for OS according to the year that the studies were published. After the subgroup analysis, there was no between-study heterogeneity. It showed that the year the study was published was a major source of heterogeneity. Because the studies were published in different years, the clinical methods they used were also different. Moreover, two of the analytical groups (Cold-II [8] and the study by Ke-Da et al. [11]) might be the source of heterogeneity. After excluding them, the heterogeneity significantly decreased. We also found that the source of heterogeneity for DFS was the study by Ke-Da. The different results may be caused by the small sample size, patient selection bias, relatively short waiting times, inappropriate WT category classification and use of an unconventional number of cyclesof chemotherapy.
According to breast cancer subtype, Gagliato et al. [14] and Chavez-MacGregor et al. [17] categorized patients into hormone receptor-positive, ERBB2-positive, and TNBC subgroups. The HRs for OS between WT $\leq 30$ days and 31-60 days in the three subgroups was extracted and analyzed. Results showed that a WT of 31-60 days had no significant impact on patients with ERBB2+ tumors or hormone receptor-positive tumors. While with TNBC, a WT 31-60 days resulted in a $26 \%$ increased risk of death. This result could be due to the rapid proliferation rate and aggressive biology of these tumors [42-44]. We did not find a statistically significant adverse effect on OS among patients with ERBB2+ tumors who had a WT 31-60 days. This might be due to the variable use of trastuzumab-based therapy and the small sample sizes in the ERBB2+ category in the two studies (Gagliato et al. [14] and Chavez-MacGregor et al. [17]).

There are still some limitations to this study. First, all the studies included in this meta-analysis are non random and retrospective. However, it is the only way to perform this type of analysis. Second, not all the prognostic factors we readjusted in our meta-analysis. Other crucial prognostic factors, such as the number of AC cycles, the dose of chemotherapeutic drugs, completion rate for $\mathrm{AC}$, HER2 status and accepting endocrine therapy or not, were not always balanced between the eligible studies. Third, we assume that the effect of WT on survival should be a log-linear relationship. However, the assumption probably didn't conform to the reality. Some studies have shown that if patients initiated AC within 12 weeks after surgery their survival was similar, and those initiating $\mathrm{AC}$ at more than 12 weeks had a significant decrease in survival

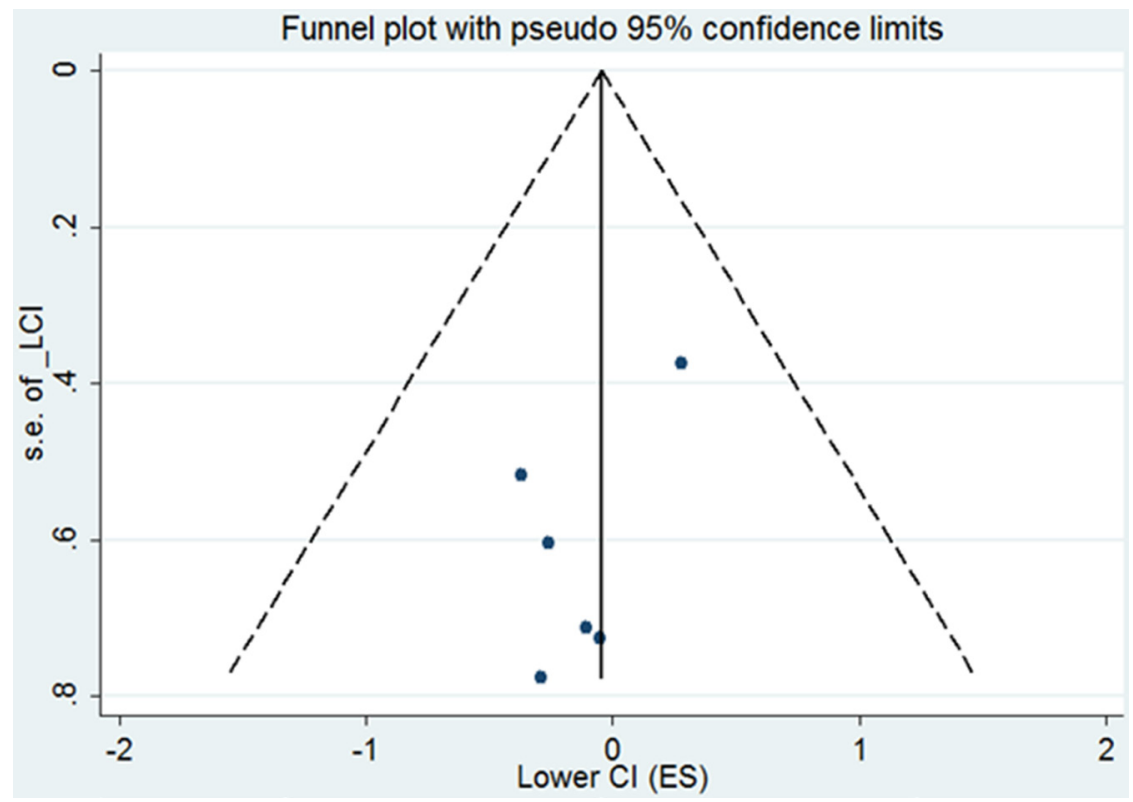

Figure 7: Funnel plot of the relationship between the hazard ratio and standard error of the log HR for overall survival in three different subtypes. The dotted line indicates the combined HR for all studies of overall survival. Filled circles represent the 6 subgroups to account for potential publication bias. 
$[16,18]$. Therefore, it may be unreliable to use the regressed summary HR across the whole time period to represent the effect of WT on survival. Moreover, the regressed summary HR is unsuitable for extrapolation outside of the time period covered by all the inclusive studies. Fourth, the effect on survival of delaying AC might be different in patients with different clinicopathological features. According to breast cancer subtype, Gagliato et al. [14] and Chavez-MacGregor et al. [17] performed subgroup analysis on hormone receptor-positive, ERBB2-positive and TNBC tumors. The results showed that a longer time to adjuvant chemotherapy may lead to worse survival in TNBC patients. But we did not find the same conclusion in hormone receptor-positive or ERBB2+ tumors. However, it is insufficient to confirm this result using only two studies, and since there is a lack of individual information, we did not have the opportunity to do comprehensive sub-analyses for all the studies included in this meta-analysis. Fifth, due to the high number of older patients in the studies by Hershman et al. [18] and Nurgaliev et al. [19], almost $25 \%$ of all the patients in our metaanalysis were older than 65 years old. It is unclear whether the age distribution of the patients in this meta-analysis and in the general breast cancer population is the same. If not, it might have a potential impact on the conclusion. Finally, most eligible studies used anthracycline-based and CMF regimens, hence it may be a problem to extrapolate the results of the meta-analysis to the current taxane era.

\section{CONCLUSIONS}

Our results found a significantly unfavorable association between a delay in the initiation of $\mathrm{AC}$ and survival of breast cancer patients, especially TNBC patients. The results recommend that the initiation of $\mathrm{AC}$ should be optimized by minimizing delay, and WTs for AC should be more strictly controlled for TNBC patients.

\section{Author contributions}

Conceived and designed the experiments: C.W., Q.H.Z. and F.F.M. Performed the experiments: Q.H.Z. and J.Q.F. Analyzed the data: J.Z. Wrote the paper: Q.H.Z. and J.Q.F. All authors reviewed the manuscript.

\section{ACKNOWLEDGMENTS}

None.

\section{CONFLICTS OF INTEREST}

The authors declare that no conflict of interest exists.

\section{FUNDING}

This study was supported by the National Natural Science Foundation ( grant No.81302320), National Key
Clinical Specialty Construction Program (grant number 201030404\#), and Sci-Tech Key Program of Fujian Province (2013Y0040 and 2016J01549).

\section{REFERENCES}

1. Ferlay J, Shin HR, Bray F, Forman D, Mathers C, Parkin DM. Estimates of worldwide burden of cancer in 2008: GLOBOCAN 2008. International journal of cancer. 2010; 127:2893-917.

2. Early Breast Cancer Trialists' Collaborative Group. Polychemotherapy for early breast cancer: an overview of the randomised trials. The Lancet. 1998; 352:930-42.

3. Peto R, Davies C, Godwin J, Gray R, Pan HC, Clarke M, Cutter D, Darby S, McGale P, Taylor C, Wang YC, Bergh J, Di Leo A, et al. comparisons between different polychemotherapy regimens for early breast cancer: metaanalyses of long-term outcome among 100,000 women in 123 randomised trials. Lancet. 2012; 379:432-44.

4. Cancer R UK. About breast cancer chemotherapy. 2011. Available at: http://wwwcancerhelporguk/type/breastcancer/treatment/chemotherapy/about-breast-cancer-chemo therapy. January 25, 2011; (accessed August 08, 11) (updated January 20, 11).

5. McCulloch P, Choy A, Martin L. association between tumour angiogenesis and tumour cell shedding into effluent venous blood during breast cancer surgery. Lancet. 1995; 346:1334-5.

6. Fedewa SA, Ward EM, Stewart AK, Edge SB. Delays in adjuvant chemotherapy treatment among patients with breast cancer are more likely in African American and Hispanic populations: a national cohort study 2004-2006. J Clin Oncol. 2010; 28:4135-41.

7. Buzdar AU, Smith TL, Powell KC, Blumenschein GR, Gehan EA. Effect of timing of initiation of adjuvant chemotherapy on disease-free survival in breast cancer. Breast Cancer Res Treat. 1982; 2:163-9.

8. Cold S, Düring M, Ewertz M, Knoop A, Møller S. Does timing of adjuvant chemotherapy influence the prognosis after early breast cancer? Results of the Danish Breast Cancer Cooperative Group (DBCG). British journal of cancer. 2005; 93:627-32.

9. Jara Sanchez C, Ruiz A, Martin M, Anton A, Munarriz B, Plazaola A, Schneider J, Martinez del Prado P, Alba E, Fernandez-Aramburo A. Influence of timing of initiation of adjuvant chemotherapy over survival in breast cancer: a negative outcome study by the Spanish Breast Cancer Research Group (GEICAM). Breast Cancer Res Treat. 2007; 101:215-23. https://doi.org/10.1007/s10549-006-9282-0.

10. Shannon C, Ashley S, Smith IE. does timing of adjuvant chemotherapy for early breast cancer influence survival? J Clin Oncol. 2003; 21:3792-7.

11. Yu KD, Fan L, Qiu LX, Ling H, Jiang YZ, Shao ZM. Influence of delayed initiation of adjuvant chemotherapy 
on breast cancer survival is subtype-dependent. Oncotarget. 2017; 8:46549-46556. https://doi.org/10.18632/oncotarget. 10551 .

12. Farolfi A, Scarpi E, Rocca A, Mangia A, Biglia N, Gianni L, Tienghi A, Valerio MR, Gasparini G, Amaducci L, Faedi M, Baldini E, Rubagotti A, et al. Time to initiation of adjuvant chemotherapy in patients with rapidly proliferating early breast cancer. Eur J Cancer. 2015; 51:1874-81. https://doi. org/10.1016/j.ejca.2015.07.003.

13. Pronzato P, Campora E, Amoroso D, Bertelli G, Botto F, Conte PF, Sertoli MR, Rosso R. Impact of administrationrelated factors on outcome of adjuvant chemotherapy for primary breast cancer. Am J Clin Oncol. 1989; 12:481-5.

14. Gagliato Dde M, Gonzalez-Angulo AM, Lei X, Theriault RL, Giordano SH, Valero V, Hortobagyi GN, ChavezMacgregor M. Clinical impact of delaying initiation of adjuvant chemotherapy in patients with breast cancer. J Clin Oncol. 2014; 32:735-44. https://doi.org/10.1200/ jco.2013.49.7693.

15. Downing A, Twelves C, Forman D, Lawrence G, Gilthorpe MS. Time to begin adjuvant chemotherapy and survival in breast cancer patients: a retrospective observational study using latent class analysis. Breast J. 2014; 20:29-36. https:// doi.org/10.1111/tbj.12209.

16. Lohrisch C, Paltiel C, Gelmon K, Speers C, Taylor S, Barnett J, Olivotto IA. Impact on survival of time from definitive surgery to initiation of adjuvant chemotherapy for early-stage breast cancer. J Clin Oncol. 2006; 24:4888-94. https://doi.org/10.1200/jco.2005.01.6089.

17. Chavez-MacGregor M, Clarke CA, Lichtensztajn DY, Giordano SH. Delayed Initiation of Adjuvant Chemotherapy Among Patients With Breast Cancer. JAMA Oncol. 2016; 2:322-9. https://doi.org/10.1001/jamaoncol.2015.3856.

18. Hershman DL, Wang X, McBride R, Jacobson JS, Grann VR, Neugut AI. Delay of adjuvant chemotherapy initiation following breast cancer surgery among elderly women. Breast Cancer Res Treat. 2006; 99:313-21. https://doi. org/10.1007/s10549-006-9206-Z.

19. Nurgalieva ZZ, Franzini L, Morgan RO, Vernon SW, Liu CC, Du XL. Impact of timing of adjuvant chemotherapy initiation and completion after surgery on racial disparities in survival among women with breast cancer. Med Oncol. 2013; 30:419. https://doi.org/10.1007/s12032-012-0419-1.

20. Altundag MK, Celik I, Ozisik Y. Is there a range of time for initiation of adjuvant chemotherapy in patients with malignancy? Ann Oncol. 2000; 11:1209.

21. Biagi JJ, Raphael MJ, Mackillop WJ, Kong W, King WD, Booth CM. Association between time to initiation of adjuvant chemotherapy and survival in colorectal cancer: a systematic review and meta-analysis. Jama. 2011; 305:2335-42. https://doi.org/10.1001/jama.2011.749.

22. Larsson SC, Orsini N, Wolk A. Vitamin B6 and risk of colorectal cancer: a meta-analysis of prospective studies.
Jama. 2010; 303:1077-83. https://doi.org/10.1001/ jama.2010.263.

23. Yu KD, Di GH, Fan L, Wu J, Hu Z, Shen ZZ, Huang W, Shao ZM. A functional polymorphism in the promoter region of GSTM1 implies a complex role for GSTM1 in breast cancer. Faseb j. 2009; 23:2274-87. https://doi. org/10.1096/fj.08-124073.

24. Yu KD, Huang S, Zhang JX, Liu GY, Shao ZM. Association between delayed initiation of adjuvant CMF or anthracycline-based chemotherapy and survival in breast cancer: a systematic review and meta-analysis. BMC Cancer. 2013; 13:240. https://doi. org/10.1186/1471-2407-13-240.

25. Berlin JA, Longnecker MP, Greenland S. Meta-analysis of epidemiologic dose-response data. Epidemiology. 1993; 4:218-28.

26. Johnson ES, Lanes SF, Wentworth CE 3rd, Satterfield MH, Abebe BL, Dicker LW. A metaregression analysis of the dose-response effect of aspirin on stroke. Arch Intern Med. 1999; 159:1248-53.

27. Higgins JP, Thompson SG. Quantifying heterogeneity in a meta-analysis. Stat Med. 2002; 21:1539-58. https://doi. org/10.1002/sim. 1186 .

28. Higgins JP, Thompson SG, Deeks JJ, Altman DG. Measuring inconsistency in meta-analyses. Bmj. 2003; 327:557-60.

29. Egger M, Davey Smith G, Schneider M, Minder C. Bias in meta-analysis detected by a simple, graphical test. Bmj. 1997; 315:629-34.

30. Colleoni M, Bonetti M, Coates AS, Castiglione-Gertsch M, Gelber RD, Price K, Rudenstam CM, Lindtner J, Collins J, Thurlimann B, Holmberg S, Veronesi A, Marini G, et al. Early start of adjuvant chemotherapy may improve treatment outcome for premenopausal breast cancer patients with tumors not expressing estrogen receptors. the international breast cancer study group. J Clin Oncol. 2000; 18:584-90.

31. Kerbrat P, Roche H, Fumoleau P, Bonneterre J, Romestaing P, Fargeot P, Namer M, Monnier A, Montcuquet P, Goudier M. Does time interval between surgery and adjuvant chemotherapy initiation modify treatment efficacy in operable, breast cancer patients? French Adjuvant Study Group (FASG) Results. J Clin Oncol. 2005; 23:660.

32. Bonadonna G, Valagussa P, Moliterni A, Zambetti M, Brambilla C. Adjuvant cyclophosphamide, methotrexate, and fluorouracil in node-positive breast cancer: the results of 20 years of follow-up. N Engl J Med. 1995; 332:901-6.

33. Henderson IC, Berry DA, Demetri GD, Cirrincione CT, Goldstein LJ, Martino S, Ingle JN, Cooper MR, Hayes DF, Tkaczuk KH, Fleming G, Holland JF, Duggan DB, et al. Improved outcomes from adding sequential paclitaxel but not from escalating doxorubicin dose in an adjuvant chemotherapy regimen for patients with node-positive primary breast cancer. J Clin Oncol. 2003; 21:976-83. 
34. Levine MN, Bramwell VH, Pritchard KI, Norris BD, Shepherd LE, Abu-Zahra H, Findlay B, Warr D, Bowman D, Myles J, Arnold A, Vandenberg T, MacKenzie R, et al. Randomized trial of intensive cyclophosphamide, epirubicin, and fluorouracil chemotherapy compared with cyclophosphamide, methotrexate, and fluorouracil in premenopausal women with node-positive breast cancer. national cancer institute of canada clinical trials group. J Clin Oncol. 1998; 16:2651-8.

35. French Adjuvant Study Group. benefit of a high-dose epirubicin regimen in adjuvant chemotherapy for nodepositive breast cancer patients with poor prognostic factors: 5-year follow-up results of french adjuvant study group 05 randomized trial. J Clin Oncol. 2001; 19:602-11.

36. Goldie JH, Coldman AJ. A mathematic model for relating the drug sensitivity of tumors to their spontaneous mutation rate. Cancer Treat Rep. 1979; 63:1727-33.

37. Fisher B, Gunduz N, Coyle J, Rudock C, Saffer E. Presence of a growth-stimulating factor in serum following primary tumor removal in mice. Cancer Res. 1989; 49:1996-2001.

38. Alkis N, Durnali AG, Arslan UY, Kocer M, Onder FO, Tokluoglu S, Celenkoglu G, Muallaoglu S, Utkan G, Ulas A, Altundag K. Optimal timing of adjuvant treatment in patients with early breast cancer. Med Oncol. 2011; 28:1255-9.

39. Brooks R, Jones S, Salmon S, Chase E, Davis S, Moon T, Giordano G, Ketchel S, Jackson R. Improved outcome with early treatment in an adjuvant breast cancer program. Proc Am Soc Clin Oncol. 1983; 110.

40. Samur M, Bozcuk H, Dalmaz G, Karaveli S, Köseoğlu F, Colak T, Pestereli E. Treatment delay in breast cancer; does it really have an impact on prognosis? Turk J Canc. 2002; 32:138-47.

41. Biagi J, Raphael M, King W, Kong W, Booth C, Mackillop W. The effect of delay in time to adjuvant chemotherapy (TTAC) on survival in breast cancer (BC): A systematic review and meta-analysis. J Clin Oncol. 2011; 29:abstr 1128.

42. Berry DA, Cirrincione C, Henderson IC, Citron ML, Budman DR, Goldstein LJ, Martino S, Perez EA, Muss HB, Norton L, Hudis C, Winer EP. Estrogen-receptor status and outcomes of modern chemotherapy for patients with nodepositive breast cancer. Jama. 2006; 295:1658-67.

43. Colleoni M, Viale G, Zahrieh D, Pruneri G, Gentilini O, Veronesi P, Gelber RD, Curigliano G, Torrisi R, Luini A, Intra M, Galimberti V, Renne G, et al. Chemotherapy is more effective in patients with breast cancer not expressing steroid hormone receptors: a study of preoperative treatment. Clin Cancer Res. 2004; 10:6622-8.

44. Foulkes WD, Smith IE, Reis-Filho JS. Triple-negative breast cancer. N Engl J Med. 2010; 363:1938-48. https:// doi.org/10.1056/NEJMra1001389. 